Geoscience and Remote Sensing, IEEE

November 2003; 41(11) : 2670- 2673

http://dx.doi.org/10.1109/TGRS.2003.817202

(C) 2005 IEEE - All Rights Reserved
Archimer http://www.ifremer.fr/docelec/ Archive Institutionnelle de l'Ifremer

\title{
A time-frequency application with the Stokes-Woodward technique
}

\section{T. Elfouhaily ${ }^{a}$, S. Guignard ${ }^{a}$, H. Branger ${ }^{a}$, D.R. Thompson ${ }^{b}$, B. Chapron ${ }^{c_{*}}$, and D. Vandemark ${ }^{d}$}

${ }^{a}$ Center National de la Recherche Scientifique, Institut de Recherche sur les Phénomènes Hors

Equilibre, Marseille, France

${ }^{\mathrm{b}}$ Univ. Applied Physics Lab., Laurel MD USA

'Département d'Océanographie Spatiale, IFREMER, France

dUniv. of New Hampshire, Ocean Process Analysis Laboratory (OPAL), Durham, NH USA

*: Corresponding author : Bertrand.Chapron@ifremer.fr

\begin{abstract}
Elfouhaily et al. (2003) generalized Woodward's theorem and applied it to the case of random signals jointly modulated in amplitude and frequency. This generalization yields a new spectral technique to estimate the amount of energy due to mode coupling without calling for higher order statistics. Two power spectra are detected; the first is related to the independent modes, and the second contains extra energy caused by mode coupling. This detection is now extended from frequency to time-frequency domain. A comparison between a wavelet transform and our timefrequency technique shows good agreement along with new insight into the time occurrence of the nonlinearities or mode coupling. An application to water surface waves is given as an example.
\end{abstract}

Keywords: Amplitude-modulation-frequency-modulation, AM-FM, Horizontal-asymmetry, Instantaneous-amplitude, Instantaneous-frequency, Mode-coupling, Nonlinear-hydrodynamicprocesses, Time-frequency-distributions, Vertical-asymmetry, Wave-wave-interaction, Wind-waves 


\title{
A Time-frequency application with the Stokes-Woodward Technique
}

\author{
T. Elfouhaily, S. Guignard, H. Branger, D.R. Thompson, B. Chapron, and D. Vandemark
}

\begin{abstract}
In a recent paper, we have generalized Woodward's theorem and applied it to the case of random signals jointly modulated in amplitude and frequency. This generalization yields a new spectral technique to estimate the amount of energy due to mode coupling without calling for higher-order statistics. Two power spectra are detected; the first is related to the independent modes and the second contains extra energy caused by mode coupling. This detection is now extended from frequency to timefrequency domain. A comparison between a wavelet transform and our time-frequency technique shows good agreement along with new insight into the time occurrence of the nonlinearities or mode coupling. Application to water surface waves is given in this letter as an example.
\end{abstract}

Index Terms-AM-FM modulations, mode coupling, instantaneous frequency, instantaneous amplitude, horizontal asymmetry, vertical asymmetry, wave-wave interaction, wind-waves, nonlinear hydrodynamic processes, time-frequency distributions.

\section{INTRODUCTION}

In a recent paper [1], we presented a new technique called the Stokes-Woodward technique that quantifies the amount of energy caused by nonlinear mode coupling. The starting point was a theorem stated by Woodward [2] which approximates the spectrum of frequency modulated signals by the probability density function of the instantaneous frequencies. A recent application of this theorem was successfully implemented in the study of delay and Doppler effects in bistatically reflected electromagnetic signals from the ocean surface [3]. We generalized this theorem in [1] to include joint amplitude and frequency modulations. A comparison was made of this new development with experimental data collected in a wind-wave tank. Two spectra were identified in addition to the traditional Fourier spectrum; the "bare" and the "dressed" spectra. The bare spectrum is obtained under no mode coupling conditions; or no wave-wave interactions in case of water waves. This can be understood as the occurrence of a family of random fundamental frequencies. The dressed spectrum, however, depicts the observable energy when mode coupling or wave-wave interactions are present, and therefore can be interpreted as the energy augmentation due to a family of random harmonics.

In the present letter, we show that the Stokes-Woodward technique can also be used to trace spectral energy in time.

T. Elfouhaily, S. Guignard, H. Branger are with Center National de la Recherche Scientifique, Institut de Recherche sur les Phénomènes Hors Equilibre, Marseille, France

D.R. Thompson is with the Johns Hopkins Univ. Applied Physics Lab., Laurel MD USA

B. Chapron is with the Département d'Océanographie Spatiale, IFREMER, France

D. Vandemark is with Univ. of New Hampshire, Ocean Process Analysis Laboratory (OPAL), Durham, NH USA
In this case, a new time-frequency technique is born, which detects wave occurrences in time. This transformation carries more information than a traditional wavelet transform. Three histograms estimated from the data enter into the study of local events. This time-frequency traceability is amenable to detect occurrences of mode coupling. Therefore, nonlinear packets can be detected in both frequency and time.

\section{A SUMMARY OF THE STOKES-WOODWARD TECHNIQUE}

\section{A. Statistical modulation}

As presented in [2], a first generalization of Woodward's theorem reduces to what we call the "bare" spectrum of a process jointly modulated in frequency and in amplitude under the condition of high modulation indices,

$$
S_{\text {bare }}(f) \approx \frac{1}{2} \int a^{2} P(a, f) \mathrm{d} a,
$$

where $P(a, f)$ is the joint probability density function of the instantaneous amplitude and frequency. This approximation is very practical and requires only the estimation of a twodimensional histogram from the available data. However, this practicality is gained at the expense of neglecting the temporal modulations of the amplitude and frequency at smaller timescales than the dominant period. In other words, the temporal modulations are slow and interpreted as random from one period to the next, while the instantaneous amplitude and frequency are assumed constant within a dominant time period. More simply stated, the high modulation index approximation in (1) is actually an exact formulation for a random process of this form

$$
\eta(t)=a \cos (\omega t+\theta)
$$

where $a, \omega$, and $\theta$ are three time-independent random variables. The amplitude $a$ and the pulsation $\omega=2 \pi f$ can be statistically dependent, while $\theta$ is a uniformly distributed phase and independent of these other variables. This requirement on the uniformity of the phase guarantees that the signal is stationary and therefore that the autocorrelation and the spectral functions are univariate. The "bare" subscript in (1) refers to the fact that the process in (2) has lost all nonlinearity or phase coupling of harmonics within one realization of the random variables.

\section{B. Temporal and statistical modulation}

The high index limit in (1) is very illustrative. The time modulation present in (1) can now be replaced by a random modulation as in (2). Let us reformulate the modulation as

$$
\eta(t)=[a+\Delta a(t)] \cos [\omega t+\Delta \phi(t)+\theta]
$$


where the time dependence is explicitly shown in addition to the implicit random dependence of all the parameters except the time variable. The key representation of our technique is in the time dependence of the modulation that can be expanded in Fourier series about the random frequency $\omega$ as

$$
\begin{aligned}
\Delta a(t) & =\alpha_{c} \cos (\omega t+\theta)+\alpha_{s} \sin (\omega t+\theta)+\ldots \\
\Delta \phi(t) & =\beta_{c} \cos (\omega t+\theta)+\beta_{s} \sin (\omega t+\theta)+\ldots
\end{aligned}
$$

The signal in (3) can be further expanded keeping only terms of linear order in the parameters $\alpha_{c}, \alpha_{s}, \beta_{c}$, and $\beta_{s}$ to find a more poignant form given by

$$
\begin{array}{rll}
\eta(t)=\lambda+ & a & \cos [\omega t+\theta]+ \\
& \alpha & \cos [2(\omega t+\theta)]+ \\
& \beta & \sin [2(\omega t+\theta)]+\ldots
\end{array}
$$

where the coefficients $\alpha$ and $\beta$ of the second harmonic terms in (5) are related to the coefficients in (4) (see [1]). We remind the reader that all parameters in (5) are random variables except the time variable. The random variables $\alpha$ and $\beta$ explain the asymmetries of the waveform with respect to the horizontal and vertical axes, respectively, and hence nonlinearities. These asymmetries appear in a random manner on the scale of the period of the wave as depicted by the random amplitude $a$ and random frequency $\omega$.

As explained in [1], the total spectrum of the signal in (5) is then what we call the dressed spectrum and it is given by

$$
S_{\text {dressed }}(f) \approx S_{\text {bare }}(f)+S_{\mathrm{NL}}(f)
$$

which is the sum of the "bare" spectrum and a nonlinear contribution to the spectral energy defined as

$S_{\mathrm{NL}}(f)=\frac{1}{2} \int \alpha^{2} P(\alpha, f / 2) \mathrm{d} \alpha+\frac{1}{2} \int \beta^{2} P(\beta, f / 2) \mathrm{d} \beta$.

We associate this excess spectrum in (7) with terms added to the bare spectrum as contributing to the energy increase at higher frequencies due to the nonlinearities or mode coupling. This energy augmentation therefore provides the difference between the bare and the dressed spectra as originally introduced and discussed in [4]. The difference between bare and dressed spectra as given in (7) can also be assimilated with the bicoherence function of phase coupling as introduced by [5] and utilized by [6]. Indeed, energy of phase coupling to second order is another manifestation of the bispectrum defined as the Fourier transform of the skewness function using a third order cumulant. More detail about the link between bispectral analysis and the Stokes-woodward technique can be found in [1].

\section{THE TIME-FREQUENCY APPLICATION}

A time-frequency application of the Stokes-Woodward technique is readily available when integration signs are dropped in the expressions of the bare (1) and the dressed (6) spectra. The time dependence will naturally reappear as frequency, amplitude, vertical and horizontal asymmetries are instantaneous

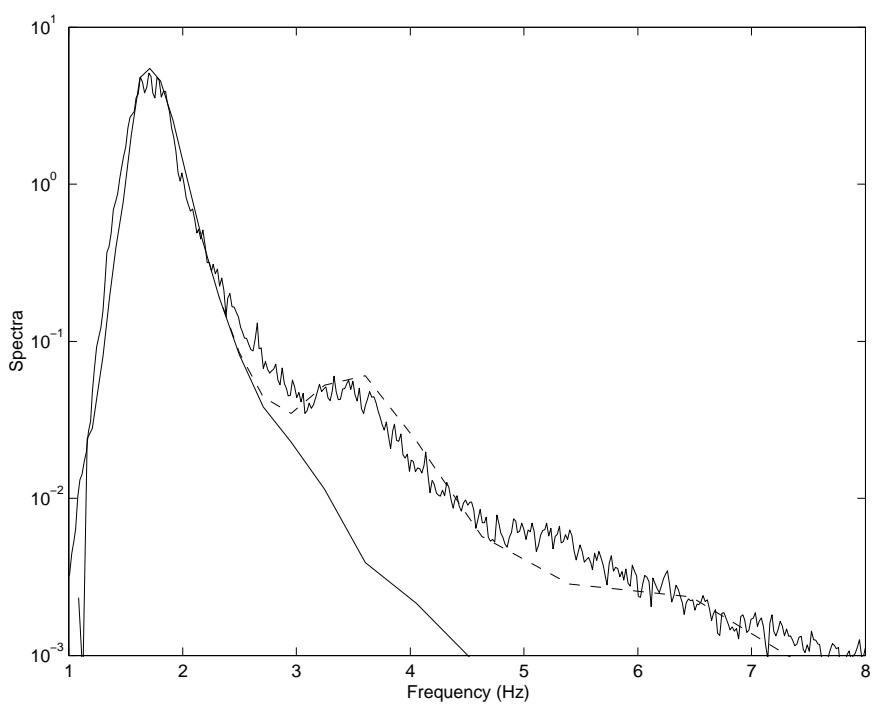

Fig. 1. The standard Fourier spectrum of the measured water surface elevation is in ragged line. The bare spectrum as defined in (1) is in solid line. The dressed spectrum in (6) is in dashed line. The effect of wave-wave coupling appears here in the additional energy provided by the dressed spectrum.

parameters and therefore time dependent. The time-frequency "bare" distribution is defined as

$$
W_{\text {bare }}(t, f)=\frac{1}{2} a(t)^{2} P(a(t), f)
$$

The nonlinear contribution is then by construction

$$
W_{N L}(t, f)=\frac{1}{2} \alpha(t)^{2} P(\alpha(t), f)+\frac{1}{2} \beta(t)^{2} P(\beta(t), f)
$$

And finally, the "dressed" spectrum is then the sum of the time-frequency energy of both the "bare" and the nonlinear contributions,

$$
W_{\text {dressed }}(t, f)=W_{\text {bare }}(t, f)+W_{N L}(t, f)
$$

The two-dimensional probability density distributions in (8) and in (9) are different and are to be estimated form the time series itself if they are not known a priori.

\section{APPLICATION ON WATER WAVES}

\section{A. Experimental data}

The time-frequency extension of the Stokes-Woodward technique is now tested using a time series generated by measuring the height of the water surface at a fixed point in a wind-wave tank. The signals were obtained from the OceanAtmosphere Interaction facility in Marseille with a capacitance wave gauge. The pool dimensions of the tank are $40 \times 3 \times 1$ meters for the length, width, and depth, respectively. The air tunnel's ceiling is $1.5 \mathrm{~m}$ above the water surface. For a detailed description of the flume-tank, the reader is referred to [7]. In order to generate highly nonlinear waves where mode coupling should be significant, we chose to sample windgenerated waves under high wind conditions with wind speeds higher than $10 \mathrm{~m} / \mathrm{s}$ with a fetch of $25 \mathrm{~m}$ but without paddle waves. Long time series were acquired at high frequency sampling. Duration and sampling rate were on the order of 30 minutes and $100 \mathrm{~Hz}$, respectively. High modulations and 
group occurrences are present in this data series. In order to estimate the instantaneous amplitude $a$, frequency $f$, height asymmetry $\alpha$ and period asymmetry $\beta$ in (5), we implemented the zero-crossing algorithm as explained in [8].

\section{B. Frequency domain}

Figure 1 shows the spectra of the time series. The lowest solid line in figure 1 is the "bare" spectrum as defined by (1) It is instructive to notice that the "bare" spectrum underestimates the energy in the tail of the measured spectrum compared to the Fourier spectrum in ragged line. This under-estimation is due to the implicit assumption in (1) that the indices of modulations are very high and therefore any nonlinearity caused by time-dependent modulations is neglected. The "dressed" spectrum (6) is shown in figure 1 by the dashed curve. This curve accounts for most of the energy present in the measured Fourier spectrum. Both vertical and horizontal asymmetries contribute to the energy at high frequencies. It therefore seems apparent that our Stokes-Woodward technique explains the energy due to mode coupling.

\section{Time-frequency domain}

The Stokes-Woodward technique can also be extended to include time variation of the spectral components shown in the previous subsection. Indeed, the time dependence of the instantaneous parameters can be exploited in (8), (9), and (10). This time frequency generalization is amenable to direct comparison with a standard time-scale distribution such as the wavelet transform. In figure 2.a the Morlet wavelet transform of our signal is shown where the $y$-axis is the inverse of scales in Hz. It is clear that the energy in the signal is mostly present about the dominant spectral peak around $1.8 \mathrm{~Hz}$. This energy however is intermittent and appears in groups or packets. These packets occur at about $1 / 10$ of the spectral peak frequency. All these features can be retrieved with the time-frequency transform of the bare spectrum and shown in panel figure 2.b. There are two major differences between the wavelet transform and our result. The first is that the wavelet is pre-determined and therefore it is signal independent. In contrast, under some conditions on the signal itself, our approach is based on the dynamical estimation of the three $2 \mathrm{D}$ histograms needed in time-frequency expressions (8) and (9). The second difference is that our transform shows the timefrequency distribution not only in terms of energy but also in terms of statistical occurrence in the total signal. As explained in our first point, the statistics of the signal are first established when estimating the 2D histograms, or the probability density distributions, and then a short segment of the data can be locally analyzed in a similar manner as the wavelet transform. The power of our transform lies in the dressed time-frequency distribution where now the occurrence of nonlinearities in time are detected. It appears that the average appearance frequency of those nonlinearities is on the order of the dominant peak frequency. This result is consistent with the fact that the signal under analysis is generated by surface water waves. When present, the occurence of the non linearities is expected to coincide with the wave crest. Our general analysis is therefore
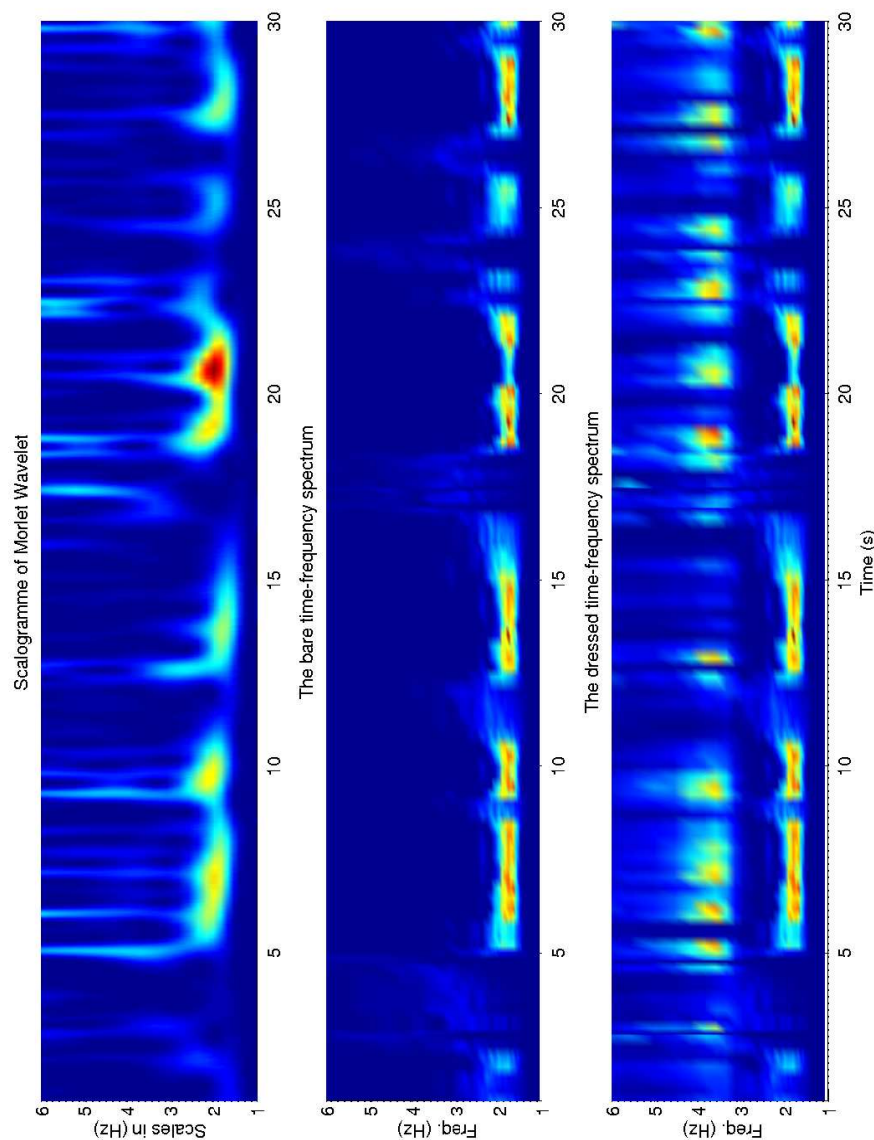

Fig. 2. Time-frequency distributions. (a) the Morlet wavelet transform. (b) the bare distribution. (c) the dressed distribution. The latter panel shows the occurrence nonlinearities.

potentially applicable to different signals with different physics behind the process being observed.

\section{CONCLUSION}

An extension to our Stokes-Woodward technique is presented where the time dependence is added to the spectral formulation developed in [1]. Our original method consisted on generalizing Woodward's theorem by including random amplitude modulations in addition to frequency modulations. The original theorem stated that a good approximation of the energy spectrum of a frequency modulated signal is the probability density function of the instantaneous frequencies when the index of modulation is high. Our generalization simply starts by including the random amplitude modulation that yields a simple spectrum expressed as a single integral over the instantaneous amplitudes and the joint distribution of amplitude and frequency as shown in (1). It is noted that this spectrum is devoid of any nonlinearity or mode coupling because over the scale of a characteristic period, the wave is considered as simply harmonic (a sine wave). Asymmetries in the wave profile must be introduced in order to capture residual energy (7) not explained by the "bare" spectrum. To account for this residual energy, we have proposed a second generalization of Woodward's theorem that utilizes a Stokeslike waveform in which all the parameters are random except 
the time variable. The total spectrum is termed the "dressed" spectrum. Both spectra were compared to a standard Fourier spectrum in figure 1. Our current extension explores the time dependence of the spectral analysis. It is shown that the bare time-frequency distribution defined in (8) and illustrated in figure 2 is in good agreement with standard Wavelet analysis. The appearance of wave groups is detected. The novel dressed time-frequency distribution as defined in (10) provides a robust estimator of the importance of mode coupling and its time dependence. We believe that our Stokes-Woodward technique has broad relevance for understanding the physics of nonlinear processes even beyond the example of surface waves discussed in the present paper.

\section{REFERENCES}

[1] T. Elfouhaily, M. Joelson, S. Guignard, H. Branger, D. Thompson, B. Chapron, and D. Vandemark, "Analysis of random nonlinear water waves: the stokes-woodward technique," submitted to Comptes Rendus de l'Académie des Sciences, 2002.

[2] P. M. Woodward, "The spectrum of random frequency modulation," Telecommunications Research Establishement, Great Malvern, Worcs., England, Technical Memorandum, 1952, 666 pages.

[3] T. Elfouhaily, D. Thompson, and L. Linstrom, "Delay-Doppler analysis of bistatically reflected signals from the ocean surface: theory and application," IEEE Trans. Geosci. and Remote Sens., vol. 40, no. 3, pp. 560-573, 2002.

[4] T. Elfouhaily, D. R. Thompson, B. Chapron, and D. Vandemark, "Weakly non-linear theory and sea state bias estimations," J. Geophys. Res., vol. 104, no. C4, pp. 7641-7647, April 151999.

[5] Y. C. Kim and E. J. Powers, "Digital bispectral analysis and its applications to nonlinear wave interactions," IEEE Trans. Plasma Sci., vol. PS-7, no. 2, pp. 120-131, 1979.

[6] M. K. Ochi and K. Ahn, "Probability distribution applicable to nongaussian random processes," Prob. Engng. Mech., vol. 9, pp. 255-264, 1994.

[7] M. Coantic and A. Favre, "Activities in and preliminary results of airsea interaction research at I.M.S.T," Adv.Geophys., vol. 16, pp. 391-405, 1974.

[8] A. Molinaro and Y. D. Sergeyev, "An efficient algorithm for the zero crossing detection in digitized measurement signal," Measurement, vol. 30, pp. 187-196, 2001. 